\title{
Awake Thyroidectomy: Safe, Effective, and Elegant
}

\author{
Noor SM Barhan ${ }^{1}$, Muhammad FN Raduan ${ }^{2}$, Nor FA Rashid ${ }^{3}$, Fauziah Ahmad ${ }^{4}$, Yahya M Aripin ${ }^{5}$
}

\begin{abstract}
Safe and margin-free resection of thyroid cancer is feasible with local or regional anesthesia. We present a case of awake thyroidectomy performed under combined local anesthesia and superficial cervical plexus block, with excellent patient satisfaction.

Keywords: Local anesthesia, Superficial cervical plexus block, Thyroidectomy.

World Journal of Endocrine Surgery (2020): 10.5005/jp-journals-10002-1308
\end{abstract}

\section{INTRODUCTION}

Thyroid surgery is customarily performed under general anesthesia. In cases which the general condition of the patient is unfavorable and the thyroid lesion is small, thyroidectomy under combined local anesthesia (LA) and superficial cervical plexus block (SCPB) may be offered.

\section{Case Description}

An 81-year-old woman, Eastern Cooperative Oncology Group (ECOG) Performance Status grade I, with coexisting hypertension, presented to us with 1 year history of a thyroid nodule which had been progressively increasing in size. Clinically, she was euthyroid and had no obstructive symptoms. On examination, a $3.5 \times 3.5 \mathrm{~cm}$ anterior neck swelling was palpable, that moved with deglutition. An ultrasound (US) of the neck showed a suspicious nodule in the left lobe close to the midline with Thyroid Imaging Reporting and Data Systems (TI-RADS) of category 5, and there was no significant cervical lymphadenopathy. Subsequent aspiration cytology reported an Atypia of Unknown Significance (AUS), Bethesda category 3. Patient was then scheduled for isthmusectomy under combined LA-SCPB in view of her age and the nodule which was small and not deeply located in the neck. Preoperatively, the patient was given counseling to ensure cooperation during surgery. The surgery was done by an experienced surgeon in the presence of assisting surgeons and an anesthetist. The patient was separated from the surgical field via a screen, but the surgeon had direct view on the patient for continuous communication and observing her facial expression. An US-guided bilateral SCPB using $6 \mathrm{~mL}$ $0.5 \%$ ropivacaine to each side was given before skin incision. Throughout the procedure, sequential LA using bupivacaine $0.5 \% 10 \mathrm{~mL}$ diluted with $10 \mathrm{~mL}$ saline injection was given in $1-2$ $\mathrm{mL}$ aliquots to each layer of dissection to ensure continuous pain control. No sedation was used in the surgery. The patient was engaged in conversation as a distraction to alleviate anxiety and maintain relaxation. Specific coaching comments by the surgeon using selective phrases were conveyed to the patient from time to time, forewarning the patient while anticipating discomfort from certain maneuver. Intraoperatively, a mixed solid-cystic nodule was noted in the isthmus which had invaded the sternothyroid muscle. The duration of the surgery was 45 minutes. Postoperative pain was minimal, and the patient was discharged home the next morning. Histopathological examination revealed papillary thyroid cancer
1-3,5 Department of Surgery, Faculty of Medicine, Universiti Teknologi MARA, Sungai Buloh Campus, Selangor, Malaysia

${ }^{4}$ Department of Anaesthesiology, Faculty of Medicine, Universiti Teknologi MARA, Sungai Buloh Campus, Selangor, Malaysia

Corresponding Author: Yahya M Aripin, Department of Surgery, Faculty of Medicine, Universiti Teknologi MARA, Sungai Buloh Campus, Selangor, Malaysia, Phone: +603 6126 5000, e-mail: mayahya3@gmail. com

How to cite this article: Barhan NSM, Raduan MFN, Rashid NFA, et al. Awake Thyroidectomy: Safe, Effective, and Elegant. World J Endoc Surg 2020;12(3):140-141.

Source of support: We thank the Dean, Faculty of Medicine, for allowing us to publish this article

Conflict of interest: None

with clear resection margins. Surveillance US of the neck 3 months after surgery showed no locoregional recurrence (Figs 1 and 2).

Part of superficial cervical plexus

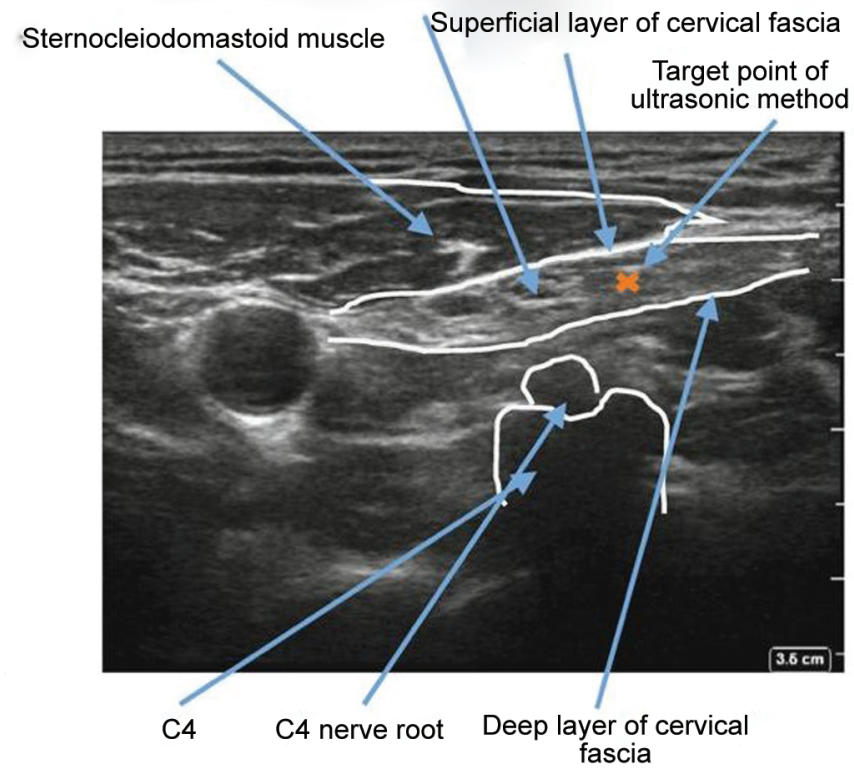

Fig. 1: The superficial cervical plexus is seen in the plane between sternocleidomastoid muscle and deep cervical fascia. The plane is injected with $6 \mathrm{~mL}$ of $0.5 \%$ ropivacaine

(0) Jaypee Brothers Medical Publishers. 2020 Open Access This article is distributed under the terms of the Creative Commons Attribution 4.0 International License (https://creativecommons.org/licenses/by-nc/4.0/), which permits unrestricted use, distribution, and non-commercial reproduction in any medium, provided you give appropriate credit to the original author(s) and the source, provide a link to the Creative Commons license, and indicate if changes were made. The Creative Commons Public Domain Dedication waiver (http://creativecommons.org/publicdomain/zero/1.0/) applies to the data made available in this article, unless otherwise stated. 


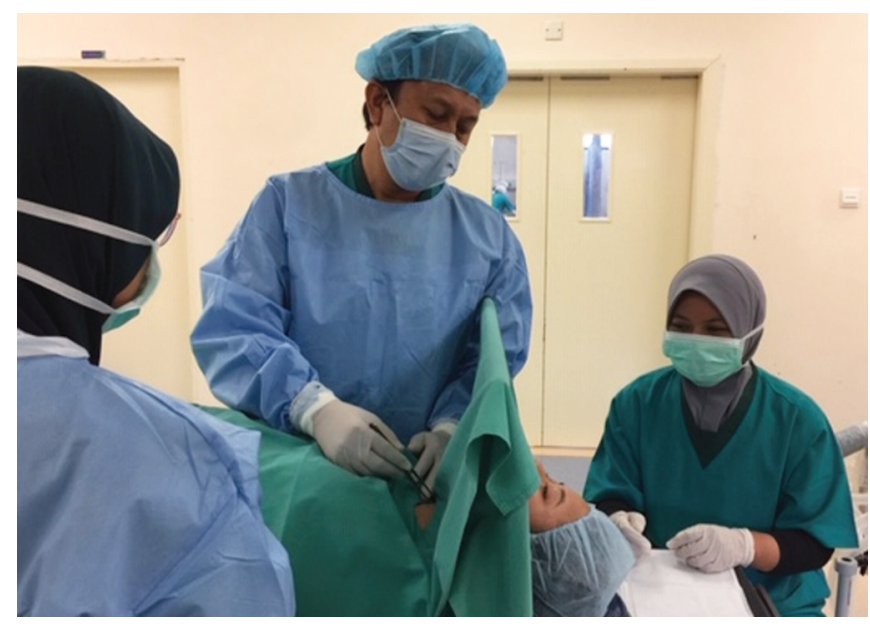

Fig. 2: A screen separates the patient from the surgical field but allows the surgeon to have direct view on the patient for communication and facial expression

\section{Discussion}

In selected cases, thyroidectomy might be performed under combined LA-SCPB. The main determining factors are the size and location of the lesion in relation to the structures in the neck. The larger the lesion and the deeper its location, the more difficult the surgery is to be expected, and thus is not a good candidate for surgery under LA-SCPB. The nature of the lesion whether benign or malignant seems not an important deciding factor since malignant nodule still can be managed by more conservative surgery and not necessarily requires more complicated or total removal of the thyroid gland. Thyroidectomy under LA-SCPB can be a worthy option in a situation when the risk of general anesthesia is morbidly intolerable such as in patients who are very frail or with compromised cardiorespiratory function.

The principles of thyroidectomy under combined LA-SCPB are similar to conventional surgery, nevertheless limited by several factors namely short half-life and limited maximum allowable volume of local anesthetics, patient's cooperation, i.e., awake and able to stay still, and availability of expertise and facilities, e.g., efficient dissection energy device. Due to this, the otherwise conventional surgery needs to be carried out in a smooth, quick, and systematic manner. One of the main vital factors for the success of awake thyroidectomy is adequate intraoperative pain control which is achievable by adequate anesthesia coupled with continuous pain monitoring which is carried out throughout the surgery via assessing patient's facial and verbal expression, in which the threshold to abandon the surgery should be kept low should continuous adequate pain control cannot be maintained.

Previous literatures had shown that thyroidectomy under LA is safe and feasible. Major complications such as permanent hypocalcemia and vocal cord paralysis were similar when compared to surgery performed under general anesthesia. The former is superior in terms of lower lung related postoperative complications. ${ }^{1}$ There is no clinically significant reduction in postoperative pain between those from SCPB to general anesthesia. ${ }^{2}$ Surgery conducted under LA also resulted in early discharge and avoidance of the post-anesthesia recovery and adverse effects ${ }^{3,4}$ This technique should be performed by an experienced surgeon to achieve an optimal patient comfort. ${ }^{5}$

\section{Conclusion}

Given proper patient selection and closed intraoperative pain monitoring and control, awake thyroidectomy can be performed with excellent patient satisfaction without compromising the safety and effectiveness of the surgery.

\section{References}

1. Hochman M, Fee WE. Thyroidectomy under local anesthesia. Arch Otolaryngol Head Neck Surg 1991;117(4):405-407. DOI: 10.1001/ archotol.1991.01870160059009.

2. Snyder SK, Roberson CR, Cummings CC, et al. Local anesthesia with monitored anesthesia care vs general anesthesia in thyroidectomy: a randomized study. Arch Surg 2006;141(2):167-173. DOI: 10.1001/ archsurg.141.2.167.

3. Lo Gerpo P. Local/regional anaesthesia for thyroidectomy: Evaluation as an outpatient procedure. Surgery 1998;124(6):975-978. DOI: 10.1016/S0039-6060(98)70037-6; discussion 978-9.

4. Mirnezami R, Sahai A, Symes A, et al. Day-case and short-stay surgery: the future for thyroidectomy? Int J Clin Pract 2007;61(7):1216-1222. DOI: 10.1111/j.1742-1241.2006.01234.x.

5. Haugen TW, Andera LN, LaMadrid AB. Awake thyroidectomy. Laryngoscope 2020;130(3):685-690. DOI: 10.1002/lary.28196. 